\title{
Comparative Evaluation of Remineralization Potential of Two Varnishes Containing CPP-ACP and Tricalcium Phosphate: An In Vitro Study
}

\author{
Venu Varma ${ }^{1}$, K Sundeep Hegde ${ }^{2}$, Sham S Bhat ${ }^{3}$, Sharan S Sargod ${ }^{4}$, HT Ajay Rao ${ }^{5}$
}

\begin{abstract}
Aim: The aim of this study is to evaluate the remineralization potential of Clinpro XT varnish containing tricalcium phosphate (TCP) and MI varnish containing casein phosphopeptide (casein phosphopeptide-amorphous calcium phosphate, CPP-ACP).

Materials and methods: Thirty premolar teeth were taken and divided into three groups. Samples were sliced mesiodistally into buccal and lingual halves using a diamond disk bur. The buccal halves of the teeth were used for the study. Artificial caries like lesions were produced and evaluated with Diagnodent. The samples in each group were treated with the respective remineralizing agent (except for the control group) at every 24 hours for 7 days and the surfaces were assessed using Diagnodent to record the values after the remineralization procedure. The Diagnodent values obtained were tabulated and statistically analyzed using one-way ANOVA and Tukey's multiple comparison tests.

Results: There was a significant difference between the values calculated before and after remineralization in all the three groups.

Conclusion: The study findings showed that MI varnish containing CPP-ACP had the highest release of fluoride as compared to the Clinpro fluoride releasing varnish.

Clinical significance: $\mathrm{MI}$ varnish is a 5\% NaF varnish containing CPP-ACP to give an exceptional fluoride varnish that releases more bioavailable fluoride, calcium, and phosphate, hence, can be used successfully in remineralization of early carious lesions. CPP-ACP can be used in the clinical practice for reversing or arresting the early carious lesions.

Keywords: Clinpro varnish, MI varnish with CPP-ACP, Tooth remineralization.

International Journal of Clinical Pediatric Dentistry (2019): 10.5005/jp-journals-10005-1629
\end{abstract}

\section{INTRODUCTION}

White-spot lesions are the earliest macroscopic evidence of enamel caries. Natural demineralization of tooth at an early stage is reversed by saliva, which contains calcium ions, phosphate ions, buffering agents, fluoride, and other substances. ${ }^{1}$ For many years, fluorides have been used for caries prevention and also for remineralization of the tooth structure. The major shortcoming of currently available toothpastes, mouth rinses, and topical applications in their ability to remineralize enamel is limited by the low concentration of calcium and phosphate ions available in saliva. This has led to the research of many new materials which can provide the oral environment with the essential elements for remineralization. Some of them are bioactive glass, casein phosphopeptide-amorphous calcium phosphate (CPP-ACP), hydroxyapatite with fluoride, etc. ${ }^{2}$

Fluoride varnish is a standard remineralizing agent developed to prolong the contact time between fluoride and the tooth surface acting as a slow releasing reservoir of fluoride. Adding calcium phosphate salts, such as TCP to the varnish, may improve the mineralization of dentin. To enhance the effect of the varnish, calcium and phosphate have been added. ${ }^{3}$

In recent years, researchers have agreed that fluoride varnishes offer an effective means of not only preventing caries but also arresting early enamel lesions.

ClinproTM XT varnish is a light-cured resin-modified glass ionomer that releases fluoride, calcium, and phosphates. The manufacturer states that ClinproTM XT varnish releases more fluoride in the first hour than conventional varnishes and releases fluoride for over 6 months. ${ }^{4}$
${ }^{1-5}$ Department of Pedodontics and Preventive Dentistry, Yenepoya
Dental College, Yenepoya University, Mangaluru, Karnataka, India
Corresponding Author: Venu Varma, Department of Pedodontics and
Preventive Dentistry, Yenepoya Dental College, Yenepoya University,
Mangaluru, Karnataka, India, Phone: +918367544666, e-mail:
drvenuvarma666@gmail.com

How to cite this article: Varma V, Hegde KS, et al. Comparative Evaluation of Remineralization Potential of Two Varnishes Containing CPP-ACP and Tricalcium Phosphate: An In Vitro Study. Int J Clin Pediatr Dent 2019;12(3):233-236.

Source of support: Nil

Conflict of interest: None

Remineralization and anticariogenic mechanism of CPP-ACP involves the incorporation of nanocomplexes into dental plaque and onto the tooth surface, which thereby acts as a calcium and phosphate reservoir.

Topically administered CPP-ACP buffers free calcium and phosphate ion activity, maintaining a state of supersaturation with respect to tooth enamel that helps prevent demineralization and facilitates remineralization on dental caries. ${ }^{1}$

Recently, more advanced fluoride varnishes with added CPPACP have been developed.

Although varnishes have been used regularly in dentistry, $\mathrm{MI}$ varnish with CPP-ACP and Clinpro XT varnish are the newer products available in the market.

There is limited research which compares Clinpro XT varnish and $\mathrm{MI}$ varnish with $\mathrm{CPP}-\mathrm{ACP}$ for the remineralization potential of carious lesion.

(c) The Author(s). 2019 Open Access This article is distributed under the terms of the Creative Commons Attribution 4.0 International License (https://creativecommons. org/licenses/by-nc/4.0/), which permits unrestricted use, distribution, and non-commercial reproduction in any medium, provided you give appropriate credit to the original author(s) and the source, provide a link to the Creative Commons license, and indicate if changes were made. The Creative Commons Public Domain Dedication waiver (http://creativecommons.org/publicdomain/zero/1.0/) applies to the data made available in this article, unless otherwise stated. 


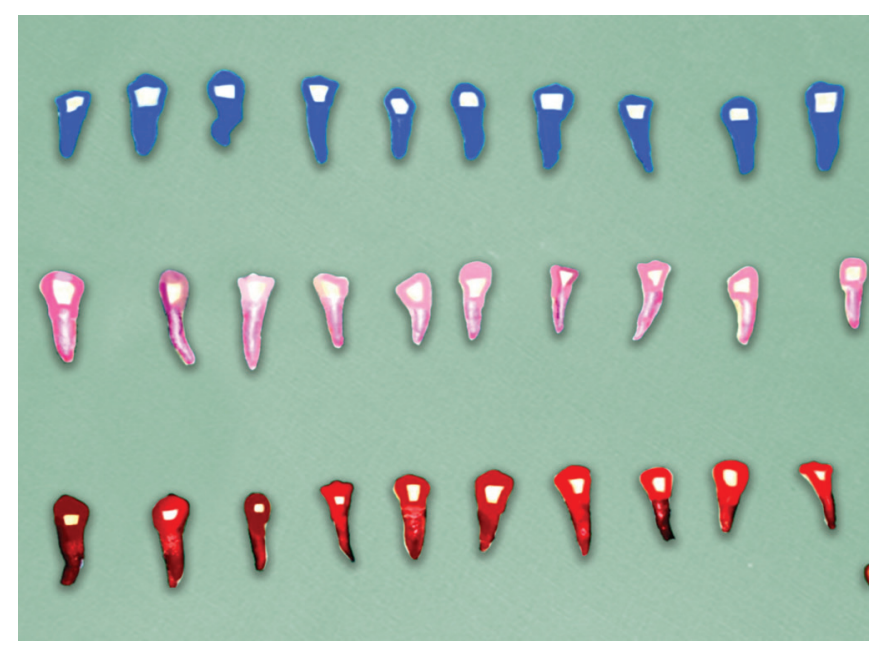

Fig. 1: Teeth divided into three groups

The present study is aimed to evaluate the remineralization potential of Clinpro XT varnish containing TCP and MI varnish containing CPP-ACP.

\section{Materials and Methods}

\section{Inclusion criteria}

Freshly extracted 30 maxillary/mandibular premolar teeth that were extracted for orthodontic reasons.

\section{Exclusion criteria}

Teeth with dental caries

Teeth with moderate to severe periodontal disease

Teeth with hypoplastic lesions

30 premolar teeth were taken and divided into three groups (Fig. 1)

The minimum sample size required is 30 teeth with an effect size of 0.6 , a level of significance $5 \%$, and a power of $80 \%$.

Group I-MI varnish with CPP-ACP

Group II-Clinpro XT varnish with durable fluoride-releasing coating (is a resin-modified glass ionomer which releases fluoride, calcium, and phosphate)

Group III-Control group

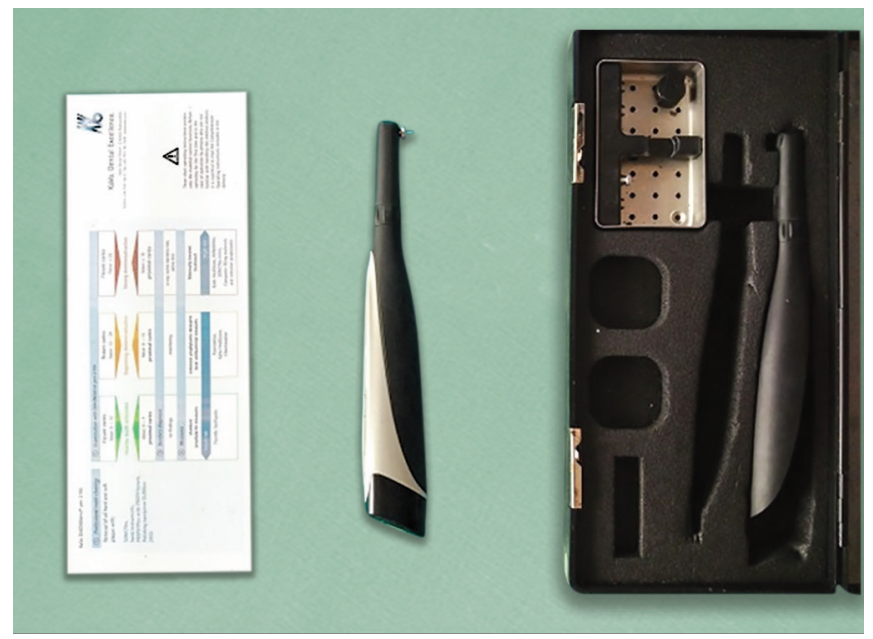

Fig. 2: Diagnodent
Teeth were stored in normal saline at room temperature until used and samples were sliced mesiodistally into buccal and lingual halves using a diamond disk bur. The buccal halves of the teeth were used for the study. The sections of the teeth were painted with an acid-resistant nail varnish exposing a window of $2 \times 2 \mathrm{~mm}$ on the center of the buccal surface of the teeth. Samples were dried with compressed air prior to application of nail varnish. Nail varnish was allowed to dry for 20 minutes and a second application of nail varnish was placed. The samples were examined using Diagnodent (Fig. 2) to assess for any surface changes present on the labial window. As recommended by the manufacturer, prior to every measurement session, the instrument was calibrated against its own ceramic standards. The labial window area was carefully scanned using the type B probe by holding the tip in close contact with the tooth surface and tilting the tip around the measuring area to collect the fluorescence from all directions. Samples showing a moment value between 3 and 7 on the digital display were selected. Samples showing a value greater than 7 were discarded and replaced by teeth having a moment value of 3-7. The baseline values of the three groups were then recorded (Fig. 3). Teeth were then immersed individually in separate plastic containers numbered from 1 to 30, each containing $4 \mathrm{~mL}$ of demineralizing solution $\left(2.2 \mathrm{mM} \mathrm{KH}_{2} \mathrm{PO}_{4}\right.$, $50 \mathrm{mM}$ acetic acid, and $2.2 \mathrm{mM} \mathrm{CaCl}_{2}$ ), incubated at $37^{\circ} \mathrm{C}$ for 96 hours to produce artificial caries like lesion. After 96 hours of incubation in the demineralizing solution, the teeth were washed with deionized water, dried with the help of an air syringe, and placed in three different clean glass containers until further evaluation.

The teeth were scanned with Diagnodent and the samples showing a moment value of 9 and above on the digital display were taken for further evaluation. This value indicated the presence of a subsurface lesion on the tooth surface. The samples were then coated with MI varnish with CPP-ACP and Clinpro varnish.

Samples in each group were treated with the respective remineralizing agent (except for the control group) at every 24 hours for 7 days, with the help of a cotton applicator tip. Each day, the samples were rubbed with the respective remineralizing agent for 4 minutes, washed with deionized water, and placed in artificial saliva. In the control group, samples were washed with only deionized water and placed in artificial saliva. Artificial saliva was changed every 24 hours just before immersion of freshly treated samples.

After seven cycles of remineralization, the surface was assessed using Diagnodent to record the values.

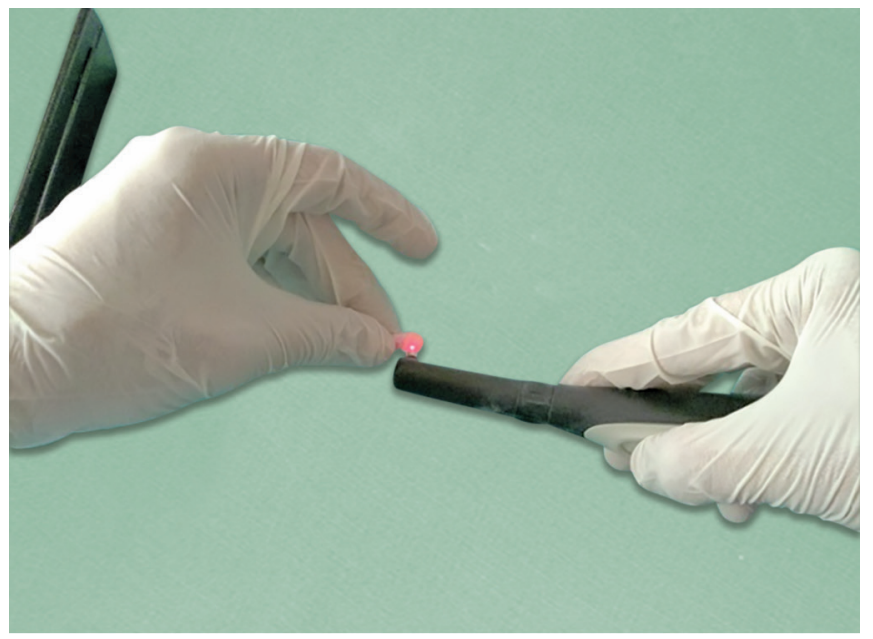

Fig. 3: Checking the values with Diagnodent 
Table 1: One-way ANOVA

\begin{tabular}{lllcll}
\hline & & $N$ & Mean & Std.deviation & p-value \\
\hline Baseline & Group I & 10 & 3.4000 & 0.51640 & 0.878 \\
& Group II & 10 & 3.4000 & 0.51640 & \\
& Control group & 10 & 3.3000 & 0.48305 & \\
& Total & 30 & 3.3667 & 0.49013 & \\
After_Demineralization & Group I & 10 & 14.6000 & 0.84327 & 0.042 \\
& Group II & 10 & 14.4000 & 1.17379 & \\
& Control group & 10 & 13.6000 & 0.51640 & \\
After_Remineralization & Total & 30 & 14.2000 & 0.96132 & \\
& Group I & 10 & 2.3000 & 0.48305 & \\
& Group II & 10 & 6.4000 & 0.69921 & \\
& Control group & 10 & 8.5000 & 0.52705 & \\
& Total & 30 & 5.7333 & 2.67728 & \\
\hline
\end{tabular}

$p<0.05$-significant

The diagnodent values obtained were tabulated and statistically analyzed using one-way ANOVA and Tukey's multiple comparison tests.

\section{Results}

The mean value at demineralization was the highest for group I (14.5), followed by group II (14.2) and then group III (13.7). There was no statistically significant difference in the mean values between the three groups. The difference between the baseline value and the demineralization value was statistically significant $(p<0.0001)$ in all the three groups.

The maximum remineralization was seen in group I (4), followed by group II (5.8) and then group III (7.5). One-way ANOVA showed that there was a significant difference among the three study groups $(p<0.0001)$ (Table 1). The multiple range test by the Tukey showed that group I (MI varnish with CPP-ACP) had significantly better remineralization than group II (Clinpro XT varnish with durable fluoride releasing coating) and group III (control group) (Fig. 4).

\section{Discussion}

In the present study, two types of remineralizing agents were used, namely MI varnish containing CPP-ACP and Clinpro fluoride releasing varnish containing TCP.

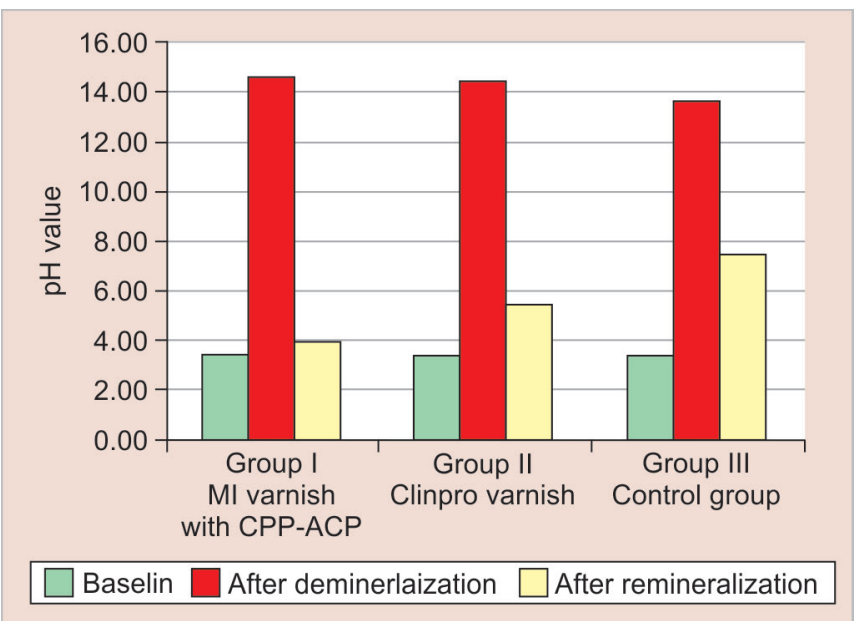

Fig. 4: Comparing three different groups: group I (MI varnish with CPPACP) showed better reminarlization than group II (Clinpro XT varnish with durable fluoride-releasing coating) and group III (control group)
Although their mechanism of action is different, the results obtained indicated that both the varnishes enhanced remineralization.

The basic mechanism of remineralization involves the diffusion of calcium and phosphate ions from saliva and other topical sources to build a hypermineralized, acid-resistant, fluorapatite like layer on the existing crystal remnants which act as remineralization nuclei. ${ }^{2}$

CPP-ACP is known to be a source of calcium and phosphate close to the sites of possible demineralization, and this is likely to inhibit demineralization, enhance remineralization, or possibly both. A possible mechanism to prevent demineralization is that casein is able to buffer plaque acid either directly or indirectly through bacterial catabolism. This agent is able to release aminoacids, and these accept protons and act as buffers. ${ }^{5}$

The recently proposed remineralization and anticariogenic mechanism of CPP-ACP involves the incorporation of nanocomplexes into dental plaque and onto the tooth surface, which thereby acts as a calcium and phosphate reservoir. ${ }^{6}$

CPP-ACP nanocomplexes act as biological calcium phosphate delivery vehicles, and are able to boost levels of bioavailable calcium and phosphate in saliva and plaque fluid without causing indiscriminate precipitation of calcium salts. This makes this material particularly effective in the remineralization of early enamel lesions and in the treatment of other types of enamel opacities.

CPP-ACP enhances the remineralization of artificially formed dentine lesions. This is consistent with a previous in situ remineralization study which showed that the CPP-ACP containing gums were able to remineralize enamel subsurface lesions. ${ }^{7}$

Though saliva has some remineralization potential, it cannot by itself increase the levels of calcium and phosphate release. For mineral deposition to occur within the body of the lesion, calcium and phosphate ions must first penetrate the surface layer of enamel. This explains why the CPP-supported metastable calcium phosphate solutions are such efficient remineralizing solutions: they are able to consume the acid generated during enamel lesion remineralization by generating more calcium and phosphate ions, including $\mathrm{CaHPO}_{4}$ thus, maintaining the high concentration gradient into the lesion.

ClinproTM XT varnish released consistently and substantially more fluoride than Fluoritop SR and Fluorprotector in the period of 6 months. This is probably due to the chemical bond between the glass ionomer of ClinproTM XT varnish and the tooth structure. Based on the resin-modified glass ionomer technology, fluoride release would be slower and extended. This study compared novel fluoride varnish (ClinproTM XT varnish) with traditional agents 
(Fluoritop SR and Fluorprotector), wherein ClinproTM XT varnish released consistently and substantially more fluoride than other tested products.

Newly marketed fluoride varnish (ClinproTM XT varnish) has significantly different fluoride release profiles compared with two conventional fluoride varnishes (Fluoritop SR and Fluorprotector). ${ }^{4}$

In a similar study, done on the ion release of calcium and fluoride containing varnishes, the authors concluded that ion release profile of MI varnish was the most promising as it had high fluoride and calcium ion release, thus, having better remineralization potential which is similar to the results of our study. ${ }^{8}$ Similar results were seen in another study done by Memarpor et al. The efficacy of CPP-ACP cream for remineralizing eroded enamel was found to be greater than fluoride toothpaste, fluoride varnish, or fTCP varnish. ${ }^{9}$

Elkassass et al. assessed enamel remineralization of different calcium-phosphate and fluoride delivery systems. Clinpro varnish presented the highest remineralization tendency with the greatest resistance for acid challenge. ${ }^{10}$ This finding is in contrast to the results of the present study, in which $\mathrm{Ml}$ varnish showed better remineralization potential than Clinpro varnish.

Shen et al. evaluated the ability of calcium phosphate and fluoride containing varnishes to inhibit enamel demineralization. MI varnish containing fluoride and CPP-ACP was superior to the other varnishes in protecting against enamel demineralization which is in accordance with the findings in the present study. ${ }^{11}$

Damyanova et al. concluded that Clinpro white varnish with TCP is effective in reducing demineralization in the subsurface layer and in improving the remineralization of surface and subsurface enamel layer. In another study, AIAmoudi et al. concluded that the use of an additive such as fTCP to a fluoride varnish significantly improves the protective ability of the varnish on primary teeth in vitro. Both the above studies ${ }^{12,13}$ are in contrast to the results of the present study.

On comparing the change in mean values from demineralization to remineralization between group II (5.8) and group I (4), the latter showed a much higher amount of remineralization. This can be attributed to the synergistic anticariogenic effects of CPP-ACP and fluoride. The fluoride ions are adsorbed onto the surface of enamel crystals, inhibiting dissolution and increasing remineralization. The softened surface lesions remineralize faster and more completely than subsurface lesions.

\section{Conclusion}

The findings of the present study showed that MI varnish containing CPP-ACP had the highest release of fluoride as compared to the Clinpro fluoride releasing varnish.

Although in the present study, maximum remineralization was seen in group I (MI varnish with CPP-ACP) followed by group II (Clinpro XT varnish with durable fluoride-releasing coating), further studies need to be done to know more about the remineralization effects of CPP-ACP and Clinpro varnish.

\section{Clinical Significance}

$\mathrm{Ml}$ varnish is a $5 \% \mathrm{NaF}$ varnish containing CPP-ACP to give an exceptional fluoride varnish that releases more bioavailable fluoride, calcium, and phosphate, hence, can be used successfully in remineralization of early carious lesions. CPP-ACP can be used in the clinical practice for reversing or arresting the early carious lesions.

\section{LIMITATIONS}

In the present study, Diagnodent was used for detecting the carious lesions. The results shown by Diagnodent are not as precise as a scanning electron microscopy, which may have affected the accuracy of our results.

\section{ACKNOWLEDGEMENT}

We convey our regards to all the staff members of the Department of Pedodontics who helped and supported us throughout the study.

\section{References}

1. Jayarajan J, Janardhanam P, et al. Efficacy of CPP-ACP and CPP-ACPF on enamel remineralization-an in vitro study using scanning electron microscope and DIAGNOdent ${ }^{\circledR}$. Indian J Dent Res 2011 Jan 1;22(1):77. DOI: 10.4103/0970-9290.80001.

2. Rajan R, Krishnan R, et al. A Polarized light microscopic study to comparatively evaluate four remineralizing agents on enamel viz CPP-ACPF, ReminPro, SHY-NM and colgate strong teeth. Int J Clin Pediatr Dent 2015 Jan;8(1):42. DOI: 10.5005/jp-journals-10005-1281.

3. Rirattanapong $P$, Vongsavan $K$, et al. Effect of fluoride varnishes containing tri-calcium phosphate sources on remineralization of initial primary enamel lesions. Southeast Asian J Trop Med Public Health 2014 Mar 1;45(2):499.

4. Virupaxi SG, Roshan NM, et al. Comparative Evaluation of Longevity of Fluoride Release From three Different Fluoride Varnishes-An In vitro Study. J Clin Diagn Res 2016 Aug;10(8):ZC33. DOI: 10.7860/ JCDR/2016/19209.8242.

5. Patil N, Choudhari S, et al. Comparative evaluation of remineralizing potential of three agents on artificially demineralized human enamel: an in vitro study. J Conserv Dent 2013 Mar 1;16(2):116. DOI: 10.4103/0972-0707.108185.

6. Gjorgievska ES, Nicholson JW. A preliminary study of enamel remineralization by dentifrices based on Recalden (CPP-ACP) and Novamin (calcium-sodium-phosphosilicate). Acta Odontol Latinoam 2009 Dec;23(3):234-239.

7. Rahiotis C, Vougiouklakis G. Effect of a CPP-ACP agent on the demineralization and remineralization of dentine in vitro. J Dent. 2007 Aug 31;35(8):695-698. DOI: 10.1016/j.jdent.2007.05.008.

8. Cochrane NJ, Shen $P$, et al. Ion release from calcium and fluoride containing dental varnishes. Aust Dent J 2014 Mar 1;59(1):100-105. DOI: $10.1111 /$ adj.12144.

9. Memarpour M, Soltanimehr E, et al. Efficacy of calcium-and fluoridecontaining materials for the remineralization of primary teeth with early enamel lesion. Microscopy research and technique $2015 \mathrm{Sep}$ 1;78(9):801-806. DOI: 10.1002/jemt.22543.

10. Elkassas D, Arafa A. Remineralizing efficacy of different calciumphosphate and fluoride based delivery vehicles on artificial caries like enamel lesions. J Dent 2014 Apr 30;42(4):466-474. DOI: 10.1016/j. jdent.2013.12.017.

11. Shen P, Bagheri R, et al. Effect of calcium phosphate addition to fluoride containing dental varnishes on enamel demineralization. Aust Dent J 2016 Sep 1;61(3):357-365. DOI: 10.1111/adj.12385.

12. Damyanova D. In Vitro Study of the Effects of Fluoride-Releasing Dental Materials on Re-Mineralization of Deciduous Teeth. IOSR J Dent Med Sci 2016 July;15(7):98-101. DOI: 10.9790/0853-1507898101.

13. AIAmoudi SA, Pani SC, et al. The effect of the addition of tricalcium phosphate to $5 \%$ sodium fluoride varnishes on the microhardness of enamel of primary teeth. Int J Dent 2013 May 28;2013:4. DOI: $10.1155 / 2013 / 486358$. 